\title{
A Mobile Computing Framework for Navigation Tasks
}

\author{
Mohammad R. Malek1,2, Mahmoud R. Delavar ${ }^{3}$ \\ and Shamsolmolook Aliabady ${ }^{2}$ \\ ${ }^{1}$ Dept. of GIS, Faculty of Geodesy and Geomatics Eng., KN Toosi University of \\ Technology, ${ }^{2}$ National Cartographic Center, ${ }^{3}$ Dept. of Surveying and Geomatics Eng., \\ University of Tehran
}

Iran

\section{Introduction}

Mobile agents and movement systems have been rapidly increased worldwide. Within the last few years, we were facing many advances in wireless communication, computer networks, location-based engines, and on-board positioning sensors. Mobile GIS as an integrating system of mobile user, wireless network, and some GIS capabilities has fostered a great interest in the GIS field [14]. Without any doubt navigation and routing could be one of the most popular GIS based solution on mobile terminals. Due to this fact the mobile GIS is defined as an area about non-geographic moving object in geographic space [22].

Although the mobile computing has been increasingly growing in the past decade, there still exist some important constraints which complicate the use of mobile GIS systems. The limited resources on the mobile computing would restrict some features that are available on the traditional computing. The resources include computational resources (e.g., processor speed and memory) user interfaces (e.g., display and pointing device), bandwidth of mobile connectivity, and energy source [3], [11], [22], and [38]. In addition, one important characteristic of such environment is frequent disconnection that is ranging from a complete to weak disconnection [11] and [44]. The traditional GIS computation methods and algorithms are not well suited for such environment. These special characteristics of mobile GIS environment make us pay more attention to this topic.

In this chapter, in order to provide a paradigm that treats with mobile objects; i.e. an automatic machine that is capable of movement in a mobile information environment; a logical framework is presented. In this framework the concept of spatial influenceability is combined with well-known formal structure; i.e. network structure. In our view, influenceability which stands for both space and time domains is a primary relation. It has some exclusive properties in the mobile information context. It can be served as a basis for context-aware mobile computing because it depends on abilities of single player or agent.

Within the framework of this chapter we attempt to apply an idea to treat moving objects in mobile GIS environment based on partitioning in space and time. The idea is, to divide space-time into small parts and find solution (e.g. collision-free paths and wayfinding procedures) recursively. In this paper, finding a path without any conflict which is so-called 
collision-free path is highlighted. It is an important task of routing and navigation. Collision-free path and its variants find applications in robot motion planning, intelligent transportation system (ITS), and any mobile autonomous navigation system. It will be concluded that Wayfinding which is a fundamental spatial activity that people experience in daily lives, could be solved by this method.

\section{Related Works}

An overview of collision detection, mathematical methods, and programming techniques to find collision-free and optimal path between two states for a single vehicle or a group of vehicles can be found in [8], [19], [40] and [18] respectively. In the field of robot motion planning potential field methods introduced by Khatib, are widely used [27]. The main attraction of potential method is its ability to speed up the optimization procedure. New researches in this area can be found as well in [2] and [18]. Path planning techniques using mixed-integer linear program were developed earlier, especially in the field of aerial vehicles navigation (see e.g. [32-33], [35-36], and [39]). The reader who wants to see more related topics is referred to [12]. In almost all works it is assumed that the moving object cruises within a fixed altitude layer, with a fixed target point, and its velocity is predefined. In addition, accessibility to up-to-date knowledge of the whole mobile agents and a global time frame are prerequisite. The lack of two last conditions in distributed mobile computing environment is a well-known fact.

A method for reducing the size of computation is computation slice [13] and [30]. The computation slicing as an extension of program slicing is useful to narrow the size of the program. It can be used as a tool in program debugging, testing, and software maintenance. Unlike a partitioning in space and time, which always exists, a distributed computation slice may not always exist [13].

Among others, two works using divide and conquer idea, called honeycomb and space-time grid, are closer to our proposal. The honeycomb model [9] focuses on temporal evolution of subdivisions of the map, called spatial partitions, and gives a formal semantics for them. This model develops to deal with map and temporal map only. The concept of space-time grid is introduced by Chon et al. [5-7]. Based upon the space-time grid, they developed a system to manage dynamically changing information. In the last work, they attempt to use the partitioning approach instead of an indexing one. This method can be used for storing and retrieving the future location of moving object.

In the previous work of the first author [25-28] a theoretical framework using Influenceability and a qualitative geometry in the mobile environment with application in the relief management was presented. This article can be considered as an empirical extension of them.

\section{Algebraic and Topological structure}

Causality is a well-known concept. There is much literature on causality, extending philosophy, physics, artificial intelligence, cognitive science and so on (e.g. [1, 16, and 40]). In our view, influenceability stands for spatial causal relation, i.e. objects must come in contact with one another; cf. [1]. Although influenceability as a primary relation does not need to prove, it has some exclusive properties which show why it is selected. Influenceability supports contextual information and can be served as a basis for context 
aware mobile computing which has attracted researchers in recent years [10] and [31]. This relation can play the role of any kind of accident and collision. It is well-known that the accident is the key parameter in most transportation systems (for example see [36]). As an example the probability of collision defines the GPS navigation integrity requirement. In addition, this model due to considering causal relation is closer to a naïve theory of motion [30].

In the relativistic physics [17] based on the postulate that the vacuum velocity of light $c$ is constant and maximum velocity, the light cone can be defined as a portion of space-time containing all locations which light signals could reach from a particular location (Figure 1). With respect to a given event, its light cone separates space-time into three parts, inside and on the future light cone, inside and on the past light cone, and elsewhere. An event $A$ can influence (influenced by) another event; $\boldsymbol{B}$; only when $\boldsymbol{B}(\boldsymbol{A})$ lies in the light cone of $\boldsymbol{A}(\boldsymbol{B})$. In a similar way, the aforementioned model can be applied for moving objects. Henceforth, a cone is describing an agent in mobile GIS environment for a fixed time interval. That means, a moving object is defined by a well-known acute cone model in space-time. This cone is formed of all possible locations that an individual could feasibly pass through or visit. The current location or apex vertex and speed of object is reported by navigational system or by prediction. The hyper surface of the cone becomes a base model for spatio-temporal relationships, and therefore enables analysis and further calculations in space-time. It also indicates fundamental topological and metric properties of space-time.

As described in Malek [25- 26], the movement modeling, are expressed in differential equation defined over a 4-dimensional space-time continuum. The assumption of a 4dimensional continuum implies the existence of 4-dimensional spatio-temporal parts. It is assumable to consider a continuous movement on a differential manifold $\boldsymbol{M}$ which represents such parts in space and time. That means every point of it has a neighborhood homeomorphic to an open set in $\boldsymbol{R}^{n}$. A path through $\boldsymbol{M}$ is the image of a continuous map from a real interval into $\boldsymbol{M}$. The homeomorphism at each point of $\boldsymbol{M}$ determines a Cartesian coordinate system $\left(\mathrm{x}_{0}, \mathrm{x}_{1}, \mathrm{x}_{2}, \mathrm{x}_{3}\right)$ over the neighborhood. The coordinate $\mathrm{x}_{0}$ is called time. In addition, we assume that the manifold $M$ can be covered by a finite union of neighborhoods. Generally speaking, this axiom gives ability to extend coordinate system to the larger area. This area shall interpret as one cell or portion of space-time. The partitioning method is application dependent. The partitioning method depends on application purposes [6] on the one hand, and limitation of the processor speed, storage capacity, bandwidth, and size of display screen on the other hand. It is important to note that the small portion of space and time in this idea is different from the geographical area covered by a Mobile Supported Station (MSS). This idea is similar to Helmert blocking in the least squares adjustment calculation [42]. 


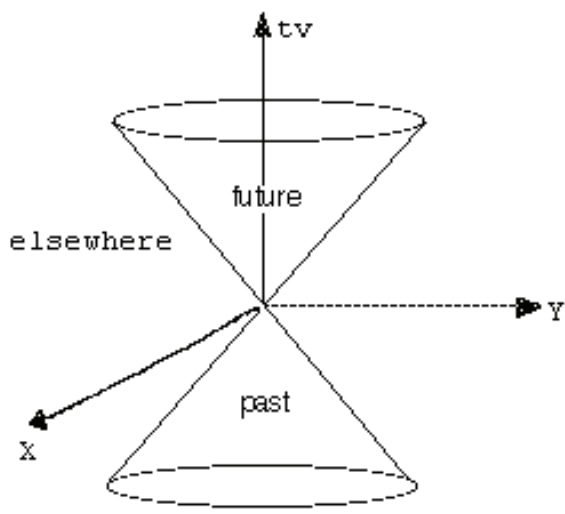

Figure 1. A cone separates space-time into 3 zones, past, future, and elsewhere

Let us take influenceability as an order relation (symbolized by $\prec$ ) be primitive relation. It is natural to postulate that influenceability is irreflexive, antisymmetric, but transitive, i.e.,

$$
(x \prec y) \wedge(y \prec z) \Rightarrow x \prec z
$$

Thus, it can play the role of 'after'.

Definition 1 (Temporal order): Let $\mathrm{x}$ and $\mathrm{y}$ be two moving objects with $t_{x}$ and $t_{y}$ corresponding temporal orders, respectively. Then,

$$
(x \prec y) \Rightarrow\left(t_{x}<t_{y}\right)
$$

Connection as a reflexive and symmetric relation [10]can be defined by influenceability as follows:

Definition 2 (Connect relation): Two moving objects $x$ and $y$ are connected if the following equation holds;

$$
(\forall x y) C(x, y):=[(x \prec y) \vee(y \prec x)] \wedge\{\neg(\exists a)[(x \prec a \prec y) \vee(y \prec a \prec x)]\}
$$

Consequently, all other exhaustive and pairwise disjoint relations in region connected calculus (RCC) [3], i.e., disconnection (DC), proper part (PP), externally connection (EC), identity (EQ), partially overlap (PO), tangential proper part (TPP), nontangential proper part (NTPP), and the inverses of the last two; TPPi and NTPPi; can be defined.

The consensus task as an acceptance of the unique framework in mobile network can not be solved in a completely asynchronous system, but as indicated by Malek [24] with the help of influenceability and partitioning concept, it can be solved. Another task in mobile network is leader election. The leader, say $a$, can be elected by the following conditions:

$$
\forall x \in\{\text { The set of moving objects }\}: a \prec x \text {. }
$$

Furthermore, some other relations can be defined, such as which termed as speed-connection (SC) and time proper overlap (TPO) (see Figure 2): 


$$
\begin{aligned}
& S C(x, y):=\neg E Q(x, y) \wedge \\
& \{[C(x, y) \wedge(\forall a b)(E C(x, a) \wedge(E C(x, b) \wedge E C(y, a) \wedge E C(y, b)] \Rightarrow C(a, b)\} \\
& T P O(x, y):=\{(x \prec y) \wedge(P O(x, y) \wedge[\forall z(S C(x, z) \Rightarrow P O(y, z))]\}
\end{aligned}
$$

a)

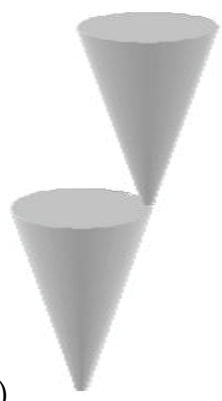

Figure 2: a) Speed-connection relation and b) Time-proper relation between two objects

As an example, team arrangement is considered. Team arrangement is an important task of any team coaching. Team arrangement in a mobile environment finds its applications not only in online problems such as Robocup or battlefield problem, but also in offline coaching. The main assumptions about mobile environment are valid in the usual coaching problem. Robocup is a well known application area of this problem. Team arrangement in such a mobile environment is a complex task in space and time. In this scenario players can be modeled with a cone based on their estimated speed and position (Figure 3).

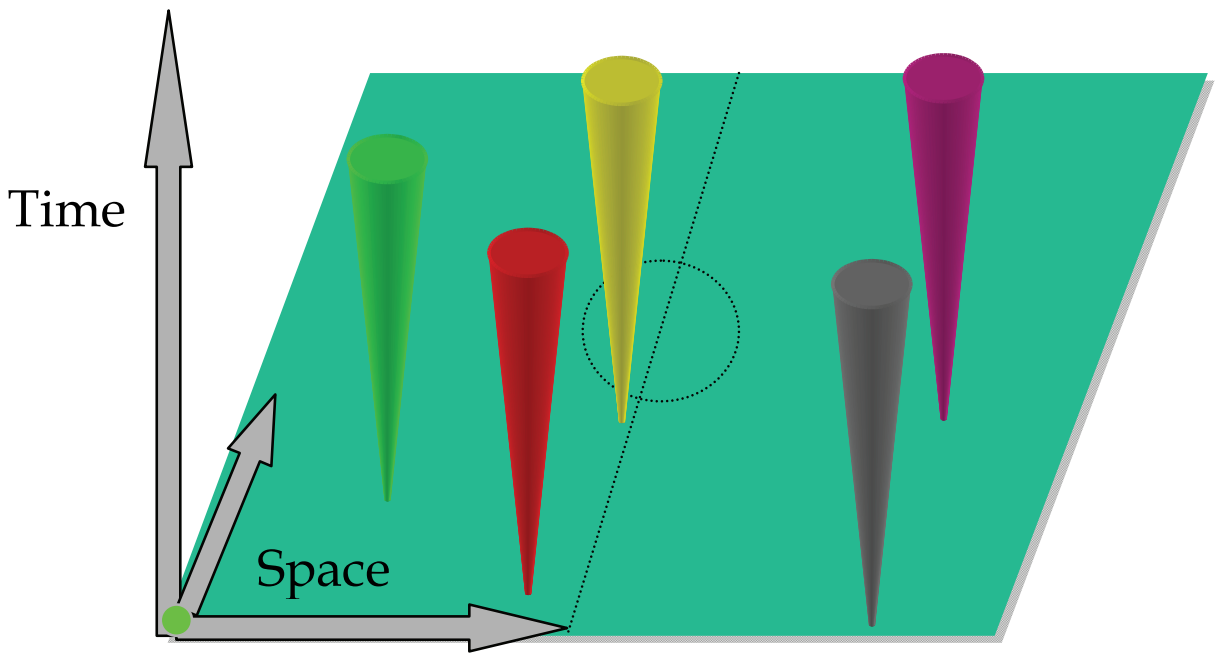

Figure 3. Robocup soccer from influenceability view point

Table 1 shows some different situations between players and their correspond relations based on our proposed model. 


\begin{tabular}{|c|c|c|}
\hline Presentation & Application & Relation \\
\hline & $\begin{array}{c}\text { Design of the defense } \\
\text { players }\end{array}$ & Players A and B overlap \\
\hline & $\begin{array}{c}\text { Man-for-man type of } \\
\text { defense }\end{array}$ & $\begin{array}{l}\text { Player A covers B } \\
\text { completely }\end{array}$ \\
\hline & $\begin{array}{l}\text { Arrangement of players to } \\
\text { minimize empty space }\end{array}$ & Externally connection \\
\hline & $\begin{array}{l}\text { Player } \mathrm{z} \text { can attack from } \\
\text { gap between } \mathrm{a} \text { and } \mathrm{b}\end{array}$ & $\begin{array}{c}C(\mathrm{a}, \mathrm{z}) \text { and } \mathrm{C}(\mathrm{b}, \mathrm{z}) \text { but } \\
\mathrm{C}(\mathrm{a}, \mathrm{b})\end{array}$ \\
\hline
\end{tabular}

Table 1. Some relations between players and their presentations

\section{Collision-Free Path}

An important task in navigation systems is to find a secure or collision-free path. A collision-free path is a route that a moving object does not have any collision or intersection with obstacles as well as other moving objects. Finding a collision-free path requires four steps, dividing the space domain into small parts, finding connected cones, computing free space, and finally solving an optimization problem. The problem discussed in this section is using a mathematical programming technique to find the optimal or near optimal collisionfree path between moving objects. The details of the other steps are left for future articles.

After partitioning space-time into space-time cells, all connected cones in space-time should be calculated. Let $[t]=\left[t_{i}, t_{i+1}\right]$ be an interval of time. The circle section of $k$ th cone at $t_{j}$; $t_{i} \leq t_{j} \leq t_{i+1}$ is denoted by $\operatorname{CIR}\left(\mathrm{O}_{k}, t_{j}\right)$, where $\mathrm{O}_{k}$ is the center point. The radius of $\operatorname{CIR}\left(\mathrm{O}_{k}, t_{j}\right)$ is calculated by speed; $\mathrm{v}_{\mathrm{k}}$;

$$
r_{k}=v_{k} \cdot\left(t_{j}-t_{i}\right)
$$

The intersection of two circles is a lens-shaped region. As it can be seen in Figure 4, the following equations can be given:

$$
\begin{gathered}
D^{R L}=\sqrt{ }\left[\left(x_{R}-x_{L}\right)^{2+}\left(y_{R}-y_{L}\right)^{2}\right] \\
a^{R L}=\left|r_{R}+r_{L}-D\right|
\end{gathered}
$$




$$
b^{R L}=r_{k}^{2}-\left(r_{k}-a\right)^{2}
$$

where $x, y$ are the coordinates of point $O$.

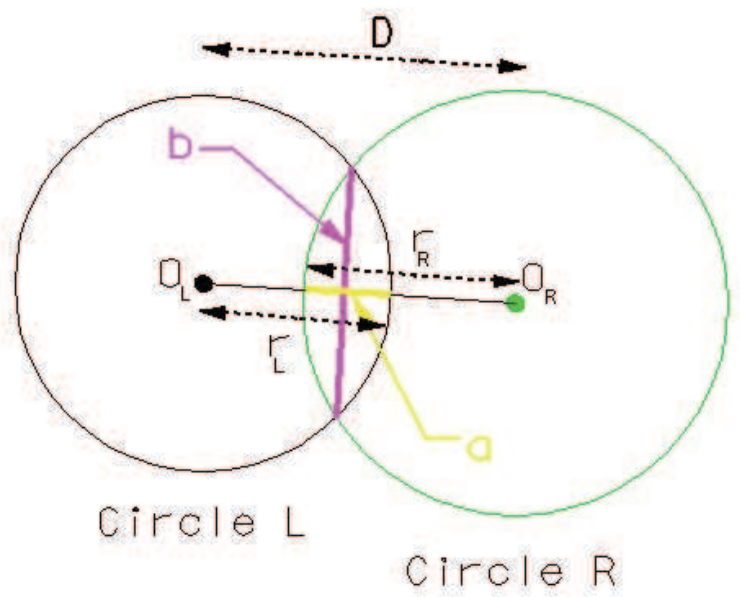

Figure 4. Intersection of two circles

Removing the barriers gives free space. It can easily be accomplished by removing all obstacles in topological structured map or by using trapezoidal map (see e.g. [8]). The trapezoidal map is an arrangement of line segments, which partitions the space into trapezoidal sections. Each trapezoidal has exactly two non-vertical boundaries and belongs to one face.

Figure 5 shows different kind of barriers. Barriers vary in size, shape and their behavior in time. The barrier (a) is constant barrier like a wall, (b) is a temporary barrier with a spatial extend, for example closing a road for a few hours, (c) is a changing size barrier in time.

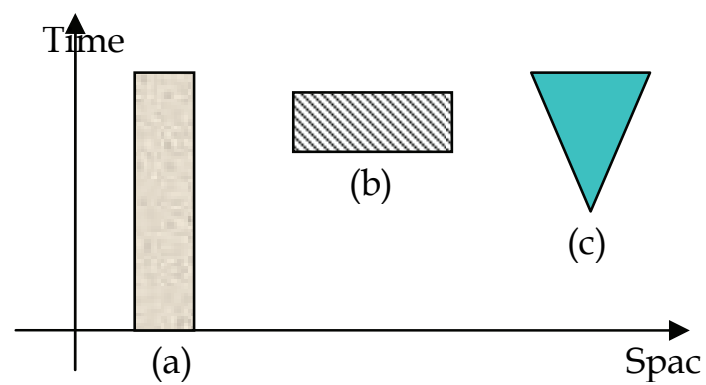

Figure 5. Different types of barriers

It will be distinguished between two network architectures, centralized and co-operative. In the centralized architecture, a control center exists which receives and sends data to moving objects. In the co-operative architecture all moving objects exchange information between themselves [21]. In the former architecture, the control variables of all nodes associates in the optimization, but in the later only variables of the active node are considered. One example 
of such optimization is used for unmanned aerial vehicles (UAV) or any other autonomously guided robots.

\subsection{Centralized Network}

As explained before, the hyper-surface of the cone indicates the fundamental topological and metrical properties of space-time. The collision-free paths, result of these cones, collectively guarantee a global collision-free route. Finding a collision-free path needs to solve the constrained optimization problem as discuss in this section.

Trying to find the new control parameters (position and speed) such that variation of parameters minimized and no collision accrued, leads to a constrained optimization problem. Consider the following model

$$
\mathbf{u}=\mathbf{u}^{*}+\mathbf{v}
$$

where $\mathbf{V} \in \mathbb{R}^{m}$ is the vector of residuals, $\mathbf{u}$ and $\mathbf{u}^{*}$ are the variables and estimated parameters, respectively. We select the sum of squares of residuals as the target function, which has to be minimized:

$$
\Phi(\mathbf{u}):=<\mathbf{v}, \mathbf{v}>\rightarrow \min
$$

The manifold $\boldsymbol{M}_{\mathrm{T}}$ can be introduced by means of target function (9) as:

$$
\begin{array}{r}
\Phi=<\mathbf{v}, \mathbf{v}>; \mathbf{u} \in \\
\boldsymbol{M}_{\mathrm{T}}:=\{\Phi \mid \mathrm{u} \in \bigcup\}
\end{array}
$$

where $\mathbb{U} \subseteq \mathbb{R}$ is an open set which includes $\mathbf{u}$ and $<_{.,}>>$indicates inner product. Minimizing $\Phi$ means finding local extreme point in target manifold [24]. In addition, having some conditions:

$$
\mathrm{G}(\mathrm{u}, \mathrm{c})=0
$$

the best looked for result must be satisfied in them. In the equation (11) the vector $\mathrm{C}$ includes the constants and n nonlinear equations are denoted by $G$. In the current problem, system equation (11) translates to

$$
D^{i j}-\left(r_{i}+r_{j}\right) \geq \varepsilon_{k} ; \forall i, j: i \neq j, k=1, \ldots, n
$$

where $\varepsilon=\varepsilon(u, S, E, \ldots)$ is a small quantity function of navigation parameters, shape and size of object, and other environment variables depending on specification of the problem domain. The total equations (9) and (12) results to a constrained optimization, which has to be solved by for instance one of 'Direct substitution', 'Constrained variation', 'Lagrange multipliers' methods [34]. The first order optimality condition leads to the system 
of nonlinear normal equations. Using the Lagrange multipliers and applying the first order optimality conditions the following system result [15]

$$
\begin{gathered}
\mathrm{u}^{*}-\left(\delta \mathrm{G}(\mathrm{u}, \mathrm{c}) / \delta \mathrm{u}^{*}\right) \cdot \mathrm{k}=\mathrm{u} \\
\mathrm{G}(\mathrm{u}, \mathrm{c})=0
\end{gathered}
$$

where $\mathrm{k}$ is Lagrange multipliers. MALEK [23-24] discussed nonlinear approaches and their geometrical interpretations to solve such problems. In a centralized architecture, $\mathrm{u}$ consists of the parameters of all objects in the net. The result of the optimization will send to the all nodes, which have influenceability relation to each other. On the contrary, in the cooperative architecture, $u$ consists of speed and position parameters of the active object and result will apply only to own itself. Through the following example the suggested method will be more clarified.

Example: Consider four vessels with the following properties:

\begin{tabular}{|l|l|l|l|}
\hline & X-Coordinate & Y-Coordinate & Speed $(\mathrm{m} / \mathrm{sec})$ \\
\hline V1 & 100 & 100 & 20 \\
\hline V2 & 200 & 200 & 16 \\
\hline V3 & -50 & -50 & 24 \\
\hline V4 & 500 & 500 & 20 \\
\hline
\end{tabular}

Table 2. Coordinate and speed at start time

By $t_{i=0} ; t_{i+1}=5$ and $\varepsilon=0$ the following table holds

\begin{tabular}{|l|l|l|l|}
\hline$r_{1}=100$ & $r_{2}=80$ & $r_{3}=120$ & $r_{4}=100$ \\
\hline
\end{tabular}

Table 3. Radius of the CIR(O,5)

By checking (7) all connected cones can be found (Table 4).

\begin{tabular}{|c|c|c|c|c|}
\hline & V1 & V2 & V3 & V4 \\
\hline V1 & - & Intersect & Intersect & Not Intersect \\
\hline V2 & & - & Not Intersect & Not Intersect \\
\hline V3 & & & - & Not Intersect \\
\hline V4 & & & & - \\
\hline
\end{tabular}

Table 4. Intersected cones

The initial position, result of system equation (13) in a centralized architecture and the final position are shown in the figure 4 , table 4 and figure 6 , respectively. 


\begin{tabular}{|l|l|l|l|}
\hline & X-Coordinate & Y-Coordinate & Speed $(\mathrm{m} / \mathrm{sec})$ \\
\hline V1 & 99.224 & 99.224 & 16.944 \\
\hline V2 & 200.6 & 200.6 & 11.73 \\
\hline V3 & -49.828 & -49.828 & 25.214 \\
\hline
\end{tabular}

Table 5. The result of constrained optimization

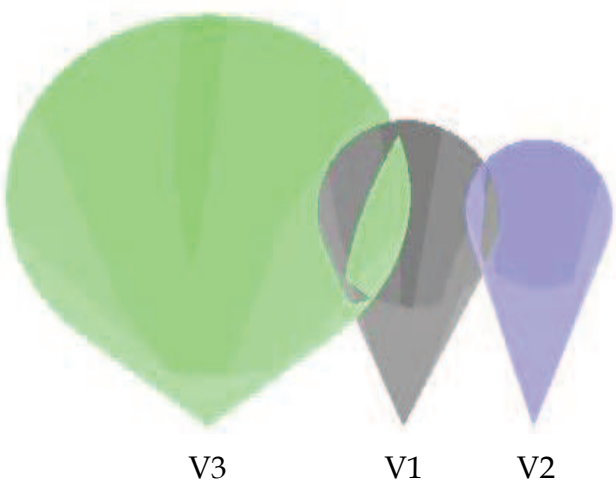

Figure 6. Four moving objects from isometric view

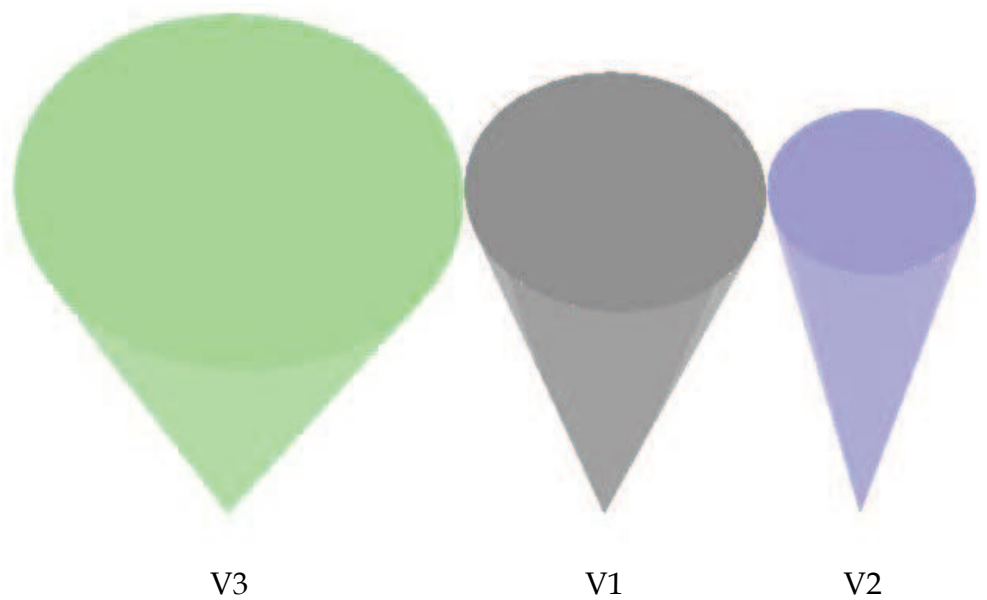

Figure 7. three moving objects after optimization from isometric view

\subsection{Co-operative Network}

Let us continue with the following scenario: A private company in order to attract more tourists to the lake "Wörthersee" in Austria provides an autonomous navigation system for their motorized small boats. Each boat equipped with a palm-top computer; using GPS for positioning; that can communicate via a wireless network. Based upon this capability, the system can play the role of online tourist guide at each part of the lake. The server sends the necessary information like current position and velocity of the other boats those are relevant 
to the mobile host. That means, the only information of the agents that have accident possibility in the current cell will be sent. As an instance, only the information of the agent number 2 will be sent to the agent number 1 in the Figure 2. This rule can be formalized by spatial influenceability relation [25]. The mobile host will send its state information like its position and velocity once they have significant changes.

At call setup an optimal route is generated. This task can be done by the fixed host. In each cell, the preliminary route is ensured that no collision occurred. It is natural to define the target function by minimizing the distance between calculated route and the optimal one. It may be named as nearest to optimal path. The method described in this part provides a minimum distance formulation (15). It is combined with the linear collision avoidance constraints [39], turn and velocity constraints, and is extended to match with partition and conquer idea.

\section{Min. $\mathbf{d}^{\mathrm{T}} \mathbf{\Sigma} \mathbf{d}$}

S.T.:

Collision avoidance, turn, and velocity conditions

d is the vector of distances between optimal state parameters and the estimated control parameters in the space-time grid of interest. Finally, the linear constraint quadratic optimization problem should be solved. This part can be run in the clients and the procedure will repeat in other parts.

\subsubsection{Collision avoidance condition}

We shall consider for simplicity of exposition of two moving objects in a two-dimensional space. The position of agent $\mathrm{p}$ at time step $\mathrm{i}$ is given by $\left(x_{p}^{i}, y_{p}^{i}\right)$ and its velocity by $\left(v_{x p}^{i}, v_{y p}^{i}\right)$, forming the elements of the state vector $\mathbf{S}_{x p}^{i}$. The real value of the state parameter is represented by an asterisk. At every time interval the corresponding surfaces; i.e. cone; of both objects must lie outside each other. It is possible to consider one object as a point and similar to classical approach taken in robot motion planning, enlarge another object with the same size. In this case, the problem becomes easier where the point should be outside of a polygon. With this trick linear conditions introduced by Schouwenaars et al. [39] can be used:

$$
\begin{aligned}
& x_{p}^{i}-x_{q}^{i} \geq d_{x}-R \cdot c_{p q 1}^{i} \text { and } \\
& x_{q}^{i}-x_{p}^{i} \geq d_{x}-R \cdot c_{p q 2}^{i} \text { and } \\
& y_{p}^{i}-y_{q}^{i} \geq d_{y}-R \cdot c_{p q 3}^{i} \text { and } \\
& y_{q}^{i}-y_{p}^{i} \geq d_{y}-R \cdot c_{p q 4}^{i} \text { and } \\
& \sum_{k=1}^{4} c_{p q k}^{i} \leq 3
\end{aligned}
$$


where $d_{l}$ is the safety distance in direction $l, c_{p q k}^{i}$ are a set of binary variables ( 0 or 1$)$ and $R$ is a positive number that is much larger than any position or velocity to be encountered in the problem.

\subsubsection{Turn and Velocity Condition}

It is possible to define other conditions to constrain the rate of turning $\left(\alpha_{\max }\right)$ and changing velocity $(\Delta)$. Turn condition can be defined with the help of coordinates. Assuming spacetime is small, linearization may apply. Other linear equations are suggested by Richards and How [36]. The velocity conditions can be derived easily as linear function from parameters.

$$
\begin{aligned}
& \frac{x_{p}^{j}-x_{p}^{i}}{y_{p}^{j}-y_{p}^{i}} \leq \alpha_{\max } \\
& v-v^{*} \leq \Delta
\end{aligned}
$$

\subsubsection{Example}

This example demonstrates that the suggested method forms an acceptable collision-free path for two boats. Figure 8 shows current locations of the boats, destinations, and space grids. The time axis is perpendicular to the space. Minimum distance optimality condition results to straight line paths to the destinations which clearly lead to a collision. In this example, the control parameters of the left vehicle are optimized.

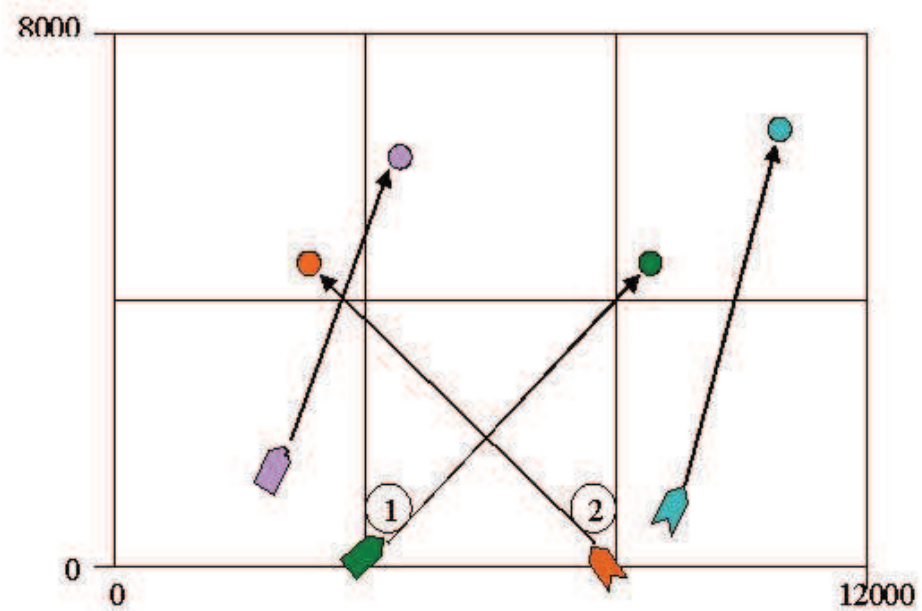

Figure 8. The trajectories of two intersecting boats. Accident will occur at fifth time interval

Let minimum speed, maximum speed, fixed time interval, and maximum deviation angle (off-route angle) be $12 \mathrm{~m} / \mathrm{s}, 30 \mathrm{~m} / \mathrm{s}, 20 \mathrm{sec}$., and 5 degrees, respectively. It can be easily seen that the approximate envelope of cones with that deviation angle is a rectangle in 2dimensional and a cylinder in 3 -dimensional space. Figure $\underline{3}$ shows the result of 
optimization with equal weight for all parameters. As can be seen, only velocity of the left boat at collision time is reduced without any significant change in direction. In order to reach a minimum time trajectory or maximum traveling with a fixed amount of money in our scenario, high weights for velocity are defined and the results are shown in the Figure9. By this method it is not necessary to assume that target point and the altitude are fixed. In each space-time cell some new object can appear. Due to linear formulation, this approach may be used in real or fast-time systems.

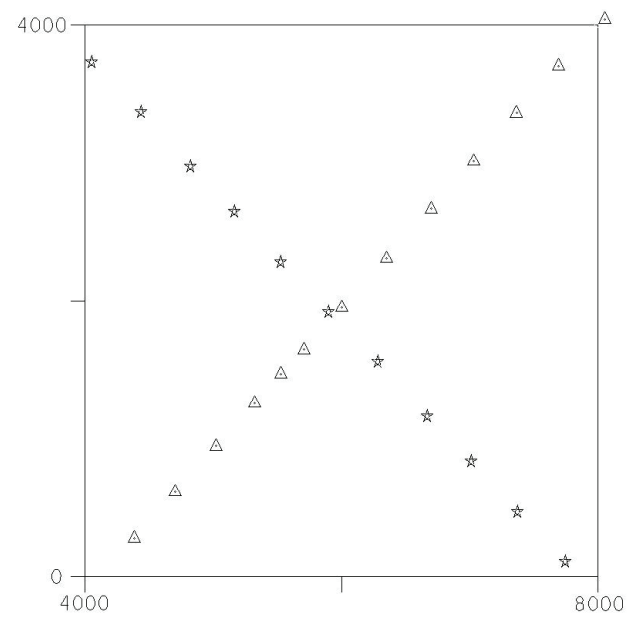

Figure 9. The designed trajectory for the left boat when all parameters are considered with equal weights
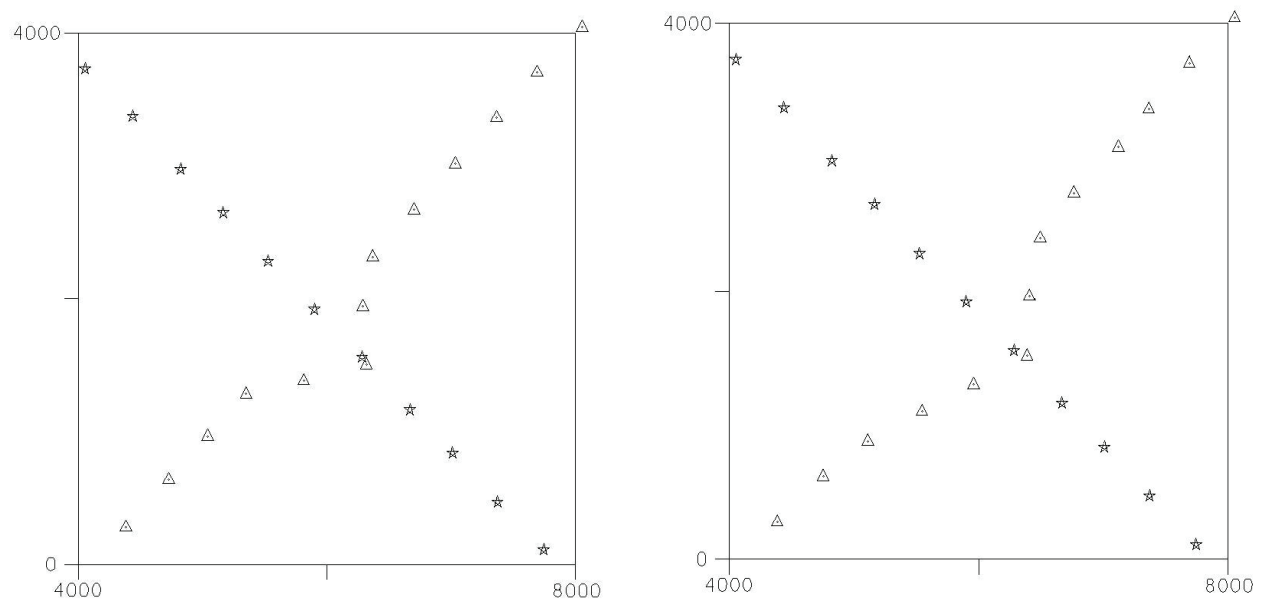

Figure 10. The designed trajectories with different turn conditions and priority of the velocity 


\section{Conclusion and Further Work}

This chapter has demonstrated that concerns to mobile GIS theory can profitably be addressed in terms of the partition and conquer idea, and influenceability relation. Of particular significance the collision-free path problem in the context of a limited resources mobile GIS environment was addressed. We have demonstrated that concerns to mobile GIS theory can be addressed profitably in terms of the partition and conquer idea. It is based on partitioning space-time into small parts, solving the problem in those small cells and connecting the results with each other to find the final result. The reasons behind are clear. The problems can be solved easier and many things are predictable at a small part of spacetime. Then, a logic-based framework for representing and reasoning about qualitative spatial relations over moving agents in space and time was derived. We provide convincing evidence of the usability of our suggested method by demonstrating how it can provide model for routing and navigation. A mathematical programming formulation has been proposed and simulated by an example to express optimal or near optimal collision-free path under the framework of such partitioning paradigm.

One important possible application of suggested methodology as our further work is mobile wayfinding services. It is based on the suggested method because wayfinding is an ordered presentation of the needed information to access an environment. It can be done in small parts as far as reaching to the desired point. A detailed uncertainty modeling for partitioning method and solving inverse problem, i.e., to determine the size and other characteristics of small parts based on the given information about needed precision, resource constraints, etc. are also among our future work.

\section{Acknowledgements}

Thanks to Prof. Frank (Technical University of Vienna) and Dr. Winter (University of Melbourne) for their comments.

\section{References}

Born, M.: Natural Philosophy of Cause and Chance, Dover Publications, New York, 1949. [1] Buchli J. (Editor): Mobile Robotics Moving Intelligence, Pro Literatur Verlag, Germany / ARS, Austria, 2006. [2]

Caduff, D. : Sketch-Based Queries In Mobile GIS Environments, Spatial Information Science and Engineering, Maine, University of Main: 114, 2002. [3]

Cohn, A. G. and Hazarika, S. M.: Qualitative Spatial Representation and Reasoning:an Overview, Fundamenta Informaticae, 43, pp. 2-32, 2001. [4]

Chon, H., Agrawal, D. and Abbadi, A. E.: Query Processing for Moving Objects with SpaceTime Grid Storage Model, Dept. of Computer Science, University of California, No: 2001-15, 2001a. [5]

Chon, H., Agrawal, D. and Abbadi, A. E.:Storage and Retrieval of Moving Objects. Proceeding of International Conference on Mobile Data Management., 2001b. [6]

Chon, H., Agrawal, D. and Abbadi, A. E.: Using Space-Time Grid for Efficient Management of Moving Objects. Proceeding of MobiDE, 2001c. [7]

De Berg, M., Van Kreveld, M., Overmars, M. and Schwarzkopf, O.: Computational GeometryAlgorithms and Application,(Berlin, Springer-Verlag, 1997. [8] 
Erwig, M. and Schneider, M., 1999, The Honeycomb Model of Spatio-temporal partitions. In Spatio-Temporal database management, (Edinburgh, Scotland, Springer), PP. 39-59. [9]

Ferscha, A. and Hoertner, H. and Kotsis, G.: Advances in Pervasive Computing, Austrian Computer Society, 2004. [10]

Forman, G. H. and Zahorjan, J., The Challenges of Mobile Computing. IEEE Computer, 27 (4), 38-47, 1994. [11]

GAMMA, http://www.cs.unc.edu/, Accessed: June 2006. [12]

Garg, V. K. and Mittal, N., Computation Slicing: Techniques and Theory. Proceeding of DISC 2001, Lisbon, Portugal, PP. 29-78, 2001. [13]

GIS-LOUNGE, http://gislounge.com/ll/mobilegis.shtml, Accessed: June 2006. [14]

Höpcke, W. : Fehlerlehre und Ausgleichrechnung, Walter de Gruyter, 1980. [15]

Lewis, D.: Causation, Journal of Philosophy, 70, pp. 556-567, 1973. [16]

Kaufmann, W. J.: Relativity and Cosmology, 1966. [17]

Kolski, S. (Editor): Mobile Robots Perception \& Navigation, Pro Literatur Verlag, Germany / ARS, Austria, 2007. [18]

Kuchar, J. K. and Yang, L. C., A Review of Conflict detection and Resolution Modeling Methods, IEEE Transactions On Intelligent Transportation Systems (December), 2000. [19]

Latombe, J.-C., Robot Motion Planning, Kluwer academic Publishers, 1991. [20]

Laurini. R., An Introduction to TeleGeoMonitoring: Problems and Potentialities, In Atkinson P. and Martin D.:Innovations in GIS, Taylor \& Francis, 2000 [21]

Li, L., Li, C. and Lin, Z., Investigation On the Concept Model Of Mobile GIS. Proceeding of Symposium on Geospatial theory, Processing and Applications, Ottawa, 2002. [22]

Malek M.R.: Nonlinear Least Squares Method from Geometric Oriented view Point, Proceedings of The First National Optimization Conference, Ferdowsi University, (1998). [23]

Malek M.R.:Nonlinear Least Squares Adjustment, Proceedings of Surveying'78 Conference, National Cartographic Center, (1999). [24]

Malek, M. R., A Logic-based Framework for Qualitative Spatial Reasoning in Mobile GIS Environment, Lecture Note in Artificial Intelligence, Vol. 3066, pp. 418- 426, Springer Verlag, 2004. [25]

Malek, M. R., Delavar, M. R. and Aliabady, S., A Logic-Based Foundation of Spatial Relationships in the Mobile GIS Environment, proceeding of the $1^{\text {st }}$ International Conference on Integrated Disaster Management, Tehran, January 2006, (in Persian). [26]

Malek, M. R. and Frank, A. U. , A Mobile Computing Approach for Navigation Purposes, Lecture Notes in Computer Science, Vol. 4295, pp. 418-426, Springer Verlag, 2006. [27]

Malek, M. R., Delavar, M. R. and Frank A.U., A Logic-Based Foundation for Spatial Relationships in Mobile GIS Environment, In: Gartner, G. and Cartwright, William; Peterson, Michael P. (Eds.): Location Based Services and Telecartography, Springer, 2007. [28]

McClosky, M.: Naive theories of motion In: Gentner D. and Stevens S. (Editors): Mental Models, Hillsdale, New Jersey, Lawrence Erlbaum, 1983. [29]

Mittal, N., Techniques for Analysing Distributed Computations, Department of Computer Science, Austin, USA, The university of Texas, 2002. [30]

Nivala, A. M. and Sarjakoski, L. T.: Need for Context-Aware Topographic Maps in Mobile Devices, In: Virrantaus, K. and Tveite, H.: ScanGIS'2003, Espoo, Finland, 2003. [31] 
Pallottino, L., Feron, E. and Bichini, A., Mixed Integer Programming for Aircraft Conflict Resolution. Proceeding of Guidance, Navigation and Control Conference, 2001. [32]

Pallottino, L., Feron, E. and Bichini, A., Conflict Resolution Problems for Air Traffic Management systems Solved with Mixed integer Programming. IEEE Transactions On Intelligent Transportation Systems, 3(1) (March), 3-11, 2002. [33]

Rao S.S. : Optimization: Theory and Applications, Wiley Eastern Ltd, (1978). [34]

Richards, A., How, J., Schouwenaars, T. and Feron, E., Plume Avoidance Maneuver Planning Using Mixed Integer Linear Programming. Proceeding of AIAA 2001, 2001. [35]

Richards, A. and How, J. P., Aircraft Trajectory Planning with Collision avoidance Using mixed Integer Linear Programming. Proceeding of American Control Conference 2002, 2002. [36]

Sang, J.: Theory and Development of GPS Integrity Monitoring System, PhD Thesis, Queensland University of Technology, 1996. [37]

Satyanarayanan, M., Fundamental Challenges in Mobile Computing. Proceeding of ACM Symposium on Principles of Distributed Computing, 1995. [38]

Schouwenaars, T., Moor, B. D., Feron, E. and How, J., Mixed Integer Programming For Multi-Vehicle Path Planning. Proceeding of European Control Conference 2001, 2001. [39]

Verma, T. S.: Causal Networks: Semantics and expressiveness, In: Sacher R. and Levitt T.S. and Kanal L.N. (ed.s):Uncertainty in Artificial Intelligence, Elsevier Science, 4, 1990. [40]

Van den Bergen, G., Collision Detection in Interactive 3D Computer Animation, Eindhoven, Eindhoven University of Technology, 1999. [41]

Wolf, H.,The Helmert block method, its origin and development, Proceeding of Second International Symposium on Problems Related to the Redefinition of North American Geodetic Networks, Arlington, PP. 319-326, 1978. [42]

Wolfson, O., Jiang, L., et al., Databases for Tracking Mobile Units in Real Time, Proceeding of Database Theory-ICDT' 99, 7th International Conference, LNCS 1540, PP. 169-186, 1999.

Zaslavsky, A. and Tari, Z., Mobile Computing: Overview and Current Status, Australian Computer Journal, 30, 1998. [43]

Zhao, Y., Vehicle Location and Navigation Systems, Boston, Artech House, 1997. [44] 


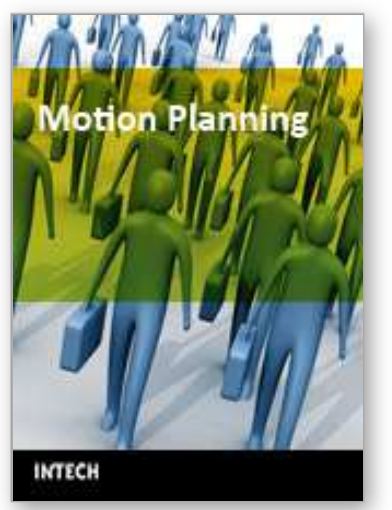

\author{
Motion Planning \\ Edited by Xing-Jian Jing
}

ISBN 978-953-7619-01-5

Hard cover, 598 pages

Publisher InTech

Published online 01, June, 2008

Published in print edition June, 2008

In this book, new results or developments from different research backgrounds and application fields are put together to provide a wide and useful viewpoint on these headed research problems mentioned above, focused on the motion planning problem of mobile ro-bots. These results cover a large range of the problems that are frequently encountered in the motion planning of mobile robots both in theoretical methods and practical applications including obstacle avoidance methods, navigation and localization techniques, environmental modelling or map building methods, and vision signal processing etc. Different methods such as potential fields, reactive behaviours, neural-fuzzy based methods, motion control methods and so on are studied. Through this book and its references, the reader will definitely be able to get a thorough overview on the current research results for this specific topic in robotics. The book is intended for the readers who are interested and active in the field of robotics and especially for those who want to study and develop their own methods in motion/path planning or control for an intelligent robotic system.

\title{
How to reference
}

In order to correctly reference this scholarly work, feel free to copy and paste the following:

Mohammad R. Malek, Mahmoud R. Delavar and Shamsolmolook Aliabady (2008). A Mobile Computing Framework for Navigation Tasks, Motion Planning, Xing-Jian Jing (Ed.), ISBN: 978-953-7619-01-5, InTech, Available from:

http://www.intechopen.com/books/motion_planning/a_mobile_computing_framework_for_navigation_tasks

\section{INTECH}

open science | open minds

\section{InTech Europe}

University Campus STeP Ri

Slavka Krautzeka 83/A

51000 Rijeka, Croatia

Phone: +385 (51) 770447

Fax: +385 (51) 686166

www.intechopen.com

\section{InTech China}

Unit 405, Office Block, Hotel Equatorial Shanghai

No.65, Yan An Road (West), Shanghai, 200040, China

中国上海市延安西路65号上海国际贵都大饭店办公楼 405 单元

Phone: +86-21-62489820

Fax: +86-21-62489821 
(C) 2008 The Author(s). Licensee IntechOpen. This chapter is distributed under the terms of the Creative Commons Attribution-NonCommercialShareAlike-3.0 License, which permits use, distribution and reproduction for non-commercial purposes, provided the original is properly cited and derivative works building on this content are distributed under the same license. 